\title{
Band-filling effects on Kondo-lattice properties
}

\author{
B. Coqblin, ${ }^{1}$ C. Lacroix,${ }^{2}$ M. A. Gusmão, ${ }^{3}$ and J. R. Iglesias ${ }^{3}$ \\ ${ }^{1}$ Laboratoire de Physique des Solides, Université Paris-Sud, Bâtiment 510, 91405 Orsay, France \\ ${ }^{2}$ Laboratoire L. Néel, CNRS, Boite Postale 166, 38042 Grenoble Cedex 09, France \\ ${ }^{3}$ Instituto de Física, Universidade Federal do Rio Grande do Sul, C.P. 15051, 91501-970 Porto Alegre, Brazil
}

(Received 8 August 2002; published 27 February 2003)

\begin{abstract}
We present theoretical results for a Kondo-lattice model with spin-1/2 localized moments, including both the intrasite Kondo coupling and an intersite antiferromagnetic exchange interaction, treated within an extended mean-field approximation. We describe here the case of a noninteger conduction-band filling for which an "exhaustion" problem arises when the number of conduction electrons is not large enough to screen all the lattice spins. This is best seen in the computed magnetic susceptibility. The Kondo temperature so obtained is different from the single-impurity one, and increases for small values of the intersite interaction, but the Kondo-effect disappears abruptly for low band filling and/or strong intersite coupling; a phase diagram is presented as a function of both parameters. A discussion of experimental results on cerium Kondo compounds is also given.
\end{abstract}

DOI: 10.1103/PhysRevB.67.064417 PACS number(s): 75.30.Mb, 71.27.+a, 75.20.Hr, 75.10. - b

\section{INTRODUCTION}

The properties of many cerium or ytterbium compounds are well accounted for by the Kondo-lattice model, where a strong competition exists between the Kondo effect and magnetic ordering arising from the Ruderman-Kittel-KasuyaYosida (RKKY) interaction between rare-earth atoms at different lattice sites. This situation is well described by the Doniach diagram, ${ }^{1}$ which gives the variation of the Néel temperature and of the Kondo temperature with increasing antiferromagnetic intrasite exchange interaction $J_{K}$ between localized spins and conduction-electron spins. If one considers the exchange Hamiltonian between localized $(\mathbf{S})$ and conduction-electron (s) spins, given by

$$
H=J_{K} \mathbf{s} \cdot \mathbf{S}
$$

usual theories of the one-impurity Kondo effect and of the RKKY interaction yield a Kondo temperature $T_{\mathrm{K} 0}$ that is proportional to $\exp \left(-1 / \rho J_{K}\right)$, and an ordering temperature (Néel or, in some cases, Curie) $T_{\mathrm{N} 0}$, proportional to $\rho J_{K}^{2}, \rho$ being the density of states for the conduction band at the Fermi energy. Thus, for small $\rho J_{K}$ values, $T_{\mathrm{N} 0}$ is larger than $T_{\mathrm{K} 0}$ and the system tends to order magnetically, with often a reduction of the magnetic moment due to the Kondo effect. On the contrary, for large $\rho J_{K}, T_{\mathrm{K} 0}$ is larger than $T_{\mathrm{N} 0}$ and the system tends to become nonmagnetic. The actual ordering temperature $T_{N}$, therefore, increases initially with increasing $\rho J_{K}$, then passes through a maximum and tends to zero at a critical value $\rho J_{K}^{c}$ corresponding to a "quantum critical point" (QCP) in the Doniach diagram. Such a behavior of $T_{N}$ has been experimentally observed with increasing pressure in many cerium compounds, such as $\mathrm{CeAl}_{2}$ (Ref. 2) or $\mathrm{CeRh}_{2} \mathrm{Si}_{2}{ }^{3}$ We also know that the Néel temperature starts from zero at a given pressure, and increases rapidly with pressure in $\mathrm{YbCu}_{2} \mathrm{Si}_{2}$ (Ref. 4) or in related ytterbium compounds, which can be considered as another test of the Doniach diagram. The one-impurity model predicts an exponential increase of the Kondo temperature with $\rho J_{K}$. This means that the Kondo temperature should increase with increasing pressure in cerium compounds and with decreasing pressure in ytterbium compounds, in good agreement with many observations. However, deviations seem to occur in some cerium compounds, such as $\mathrm{CeRh}_{2} \mathrm{Si}_{2}$ (Ref. 3) or $\mathrm{CeRu}_{2} \mathrm{Ge}_{2},{ }^{5,6}$ where the actual Kondo temperature observed in a lattice can be significantly different from the one derived for the singleimpurity case. Thus, in order to account for such an effect, we have treated in a previous paper ${ }^{7}$ the Kondo-lattice model with both intrasite Kondo exchange and intersite antiferromagnetic exchange interactions, for a half-filled conduction band (corresponding to a number of conduction electrons $n$ $=1$ ). We employed a mean-field approximation with two correlators, $\lambda_{i \sigma}$, describing the intrasite Kondo correlation, and $\Gamma_{i j \sigma}$, representing an intersite correlation between two neighboring moments. We have shown that the enhancement of the intersite exchange interaction tends to decrease the Kondo temperature $T_{K}$ for the lattice with respect to the oneimpurity Kondo temperature $T_{\mathrm{K} 0}$, and to suppress the Kondo effect for large values of the intersite exchange interaction parameter. ${ }^{7}$ So, this model can account for the pressure dependence of $T_{K}$ observed in $\mathrm{CeRh}_{2} \mathrm{Si}_{2},{ }^{3} \mathrm{CeRu}_{2} \mathrm{Ge}_{2},{ }^{5,6}$ or more recently ${ }^{8} \mathrm{Ce}_{2} \mathrm{Rh}_{3} \mathrm{Ge}_{5}$.

The above mentioned model also yields a correlation temperature $T_{\text {cor }}$, below which short-range magnetic correlations between neighboring cerium atoms occur. For sufficiently large values of the intersite exchange, $T_{\text {cor }}$ is higher than the Kondo temperature. This result is in agreement with the experimental observation by neutron diffraction experiments of such short-range magnetic correlations in single crystals of $\mathrm{CeCu}_{6},{ }^{9} \mathrm{CeInCu}_{2},{ }^{10} \mathrm{CeRu}_{2} \mathrm{Si}_{2},{ }^{9,11,12}$ or $\mathrm{Ce}_{1-x} \mathrm{La}_{x} \mathrm{Ru}_{2} \mathrm{Si}_{2}$ (Refs. 11-14) at low temperatures. The experimentally observed temperature $T_{\text {cor }}$ is clearly larger than the Kondo temperature $T_{K}: T_{\text {cor }} \sim 60-70 \mathrm{~K}$ and $T_{K} \sim 20 \mathrm{~K}$ in $\mathrm{CeRu}_{2} \mathrm{Si}_{2} ;{ }^{9,11,12} T_{\text {cor }} \sim 10 \mathrm{~K}$ and $T_{K} \sim 5 \mathrm{~K}$ in $\mathrm{CeCu}_{6}{ }^{9}$

Most Kondo-lattice models have been studied for the case of a half-filled conduction band, corresponding to a number of conduction electrons, $n$, equal to the number $n_{f}=1$ of $f$ electrons. However, when $n<1$, an "exhaustion" problem arises, which means that there are not enough conduction 
electrons to screen all the localized spins and, as a consequence, the Kondo temperature decreases. ${ }^{15,16}$ The stability of the Kondo effect when $n$ decreases has been recently studied, ${ }^{17}$ and also the temperature dependence of the magnetic susceptibility has been computed for different values of $n,{ }^{18}$ with the Hamiltonian including an intersite exchange interaction $J_{H}$ between the localized spins, treated as independent of $J_{K}$. The results showed that a reduction of $n$ and/or an enhancement of $J_{H}$ tend to suppress the Kondo effect. In particular, the analytical calculation at $T=0$ gave $\lambda^{2}$ proportional to $n$ in the case of $J_{H}=0 .{ }^{19}$

Early studies of the Kondo-lattice model have considered only the intrasite Kondo interaction. ${ }^{20-22}$ In particular, Continentino et al. ${ }^{21}$ using scaling theory, have found a coherence temperature increasing above the QCP. Recently, Burdin et al., ${ }^{23,24}$ using the slave-boson method in the exhaustion limit, have obtained two different energy scales: the single-impurity Kondo temperature $T_{K}$, which corresponds to the onset of local singlet formation, and the zerotemperature energy gain, $T^{*}$, related to the coherent Kondo effect. Thus, the low-temperature energy scale is not equal to the high-temperature one, although there is only one meanfield parameter in the theory. ${ }^{23,24}$ It has been shown that the ratio $T^{*} / T_{K}$ is much smaller than 1 , and depends only on the band filling factor. In the case of a rectangular density of states for the conduction band, $T_{K}$ vanishes as $n^{1 / 2}$ and the coherence temperature as $n$ in the limit $n \rightarrow 0$. However, both $T_{K}$ and $T^{*}$ where found to exhibit the same exponential behavior $\exp \left(-2 D / J_{K}\right)$, in contrast with the formula established by Nozières, ${ }^{16}$ who found $T^{*} \sim T_{K}^{2} / D$. The effect of a small number of conduction electrons has been also studied within both the Kondo-lattice and the Anderson-lattice models. ${ }^{25-28}$

The purpose of the present paper is to further develop the study of conduction-band filling effects within the Kondolattice model. Besides previous work cited above, ${ }^{7,18,19}$ a first brief account has been already given, ${ }^{29}$ but we will present here both numerical results and new analytical expressions for some specific limits, as well as a detailed discussion of band-filling effects.

\section{THE THEORETICAL MODEL}

As it was mentioned above, the Kondo-lattice model is often studied with only the intrasite Kondo interaction. In principle, both the RKKY magnetic interaction and the Kondo effect are consequences of the intrasite exchange term. However, the RKKY interaction is perturbative in $J_{K}$ while the Kondo effect is not. ${ }^{25}$ When dealing with approximation schemes, it is hard to obtain both effects starting with the intrasite interaction alone. For this reason, an explicit intersite exchange interaction is usually included in the model Hamiltonian. ${ }^{7,25,30}$

The proposed Hamiltonian of the system is, therefore,

$$
H=\sum_{\mathbf{k} \sigma} \varepsilon_{\mathbf{k}} n_{\mathbf{k} \sigma}^{c}+J_{K} \sum_{i} \mathbf{s}_{i} \cdot \mathbf{S}_{i}+J_{H} \sum_{\langle i j\rangle} \mathbf{S}_{i} \cdot \mathbf{S}_{j},
$$

where $\varepsilon_{\mathbf{k}}$ is the energy of the conduction band, $J_{K}$ is the Kondo coupling between a localized spin $\mathbf{S}_{i}$ and the spin $\mathbf{s}_{i}$ of a conduction electron at the same site, and $J_{H}$ is the interaction between nearest-neighboring localized spins. Assuming spin-1/2 localized moments, we represented them by a zero-width $f$ band with one electron per site, while the conduction band has width $2 D$ and a constant density of states. We choose $J_{K}$ and $J_{H}$ to be positive, implying that both local and intersite interactions are antiferromagnetic, as is the case in most cerium compounds.

We now write the spin operators in fermionic representation:

$$
\begin{gathered}
s_{i}^{z}=\frac{1}{2}\left(n_{i \uparrow}^{c}-n_{i \downarrow}^{c}\right), \quad S_{i}^{z}=\frac{1}{2}\left(n_{i \uparrow}^{f}-n_{i \downarrow}^{f}\right), \\
s_{i}^{+}=c_{i \uparrow}^{\dagger} c_{i \downarrow}, \quad S_{i}^{+}=f_{i \uparrow}^{\dagger} f_{i \downarrow}, \\
s_{i}^{-}=c_{i \downarrow}^{\dagger} c_{i \uparrow}, \quad S_{i}^{-}=f_{i \downarrow}^{\dagger} f_{i \uparrow},
\end{gathered}
$$

remembering that we have a constraint of single occupancy of the $f$ level at all sites, $n_{i}^{f}=1$.

In order to discuss the Kondo effect and magnetic correlations we define the operators

$$
\lambda_{i \sigma} \equiv c_{i \sigma}^{\dagger} f_{i \sigma}, \quad \Gamma_{i j \sigma} \equiv f_{i \sigma}^{\dagger} f_{j \sigma},
$$

where $\lambda_{i \sigma}$ describes the intrasite Kondo correlation, and $\Gamma_{i j \sigma}$ represents an intersite correlation between two neighboring atoms. This is so because the on-site spin-spin correlation $\left\langle\mathbf{s}_{i} \cdot \mathbf{S}_{i}\right\rangle$ can be written in terms of the average $\left\langle\lambda_{i \sigma} \lambda_{i,-\sigma}\right\rangle$, and similarly for the intersite correlation using $\Gamma_{i j \sigma}$. With this notation we perform an extended mean-field approximation, introduced by Coleman and Andrei ${ }^{30}$ and presented in full detail in Ref. 7. Considering translational invariance, and taking into account that there is no breakdown of spin symmetry, i.e., no magnetic states, we can write $\lambda=\left\langle\lambda_{i \sigma}\right\rangle$ for all sites, and $\Gamma=\left\langle\Gamma_{i j \sigma}\right\rangle$ for nearest-neighboring sites and zero otherwise. In this way we obtain a mean-field Hamiltonian that takes the form of a hybridized two-band system:

$$
\begin{aligned}
H^{\mathrm{MF}}= & \sum_{\mathbf{k} \sigma} \varepsilon_{\mathbf{k}} n_{\mathbf{k} \sigma}^{c}+E_{0} \sum_{i}\left(\sum_{\sigma} n_{i \sigma}^{f}-1\right)-J_{K} \lambda \sum_{i \sigma}\left(c_{i \sigma}^{\dagger} f_{i \sigma}\right. \\
& \left.+f_{i \sigma}^{\dagger} c_{i \sigma}\right)+\bar{E}_{K}-J_{H} \Gamma \sum_{\langle i j\rangle_{\sigma}}\left(f_{i \sigma}^{\dagger} f_{j \sigma}+f_{j \sigma}^{\dagger} f_{i \sigma}\right)+\bar{E}_{H},
\end{aligned}
$$

with

$$
\bar{E}_{K}=2 N J_{K} \lambda^{2}, \quad \bar{E}_{H}=z N J_{H} \Gamma^{2},
$$

$N$ being the total number of lattice sites. We have introduced a term depending on the Lagrange multiplier $E_{0}$ in order to impose the constraint $\Sigma_{i}\left(n_{i}^{f}-1\right)=0$. This is a weak form of the original constraint $n_{i}^{f}=1$.

After performing this approximation, one deals with a one-electron Hamiltonian representing two hybridized bands: the conduction band of width $2 D$ and the $f$ band of effective band width $2 B D$, with $B$ given by

$$
B=-z J_{H} \Gamma / D,
$$


$z$ being the number of nearest neighbors of a site, while the magnitude of the hybridization gap is directly related to $\lambda^{2}$. This quantity is also a measure of the Kondo effect, as the Kondo correlation function $\left\langle\mathbf{s}_{i} \cdot \mathbf{S}_{i}\right\rangle$ is proportional to $\lambda^{2}$.

The Hamiltonian (5), leaving aside the constant terms, is easily diagonalized, and the resulting energies of the two new hybrid bands are

$$
E_{\mathbf{k}}^{ \pm}=\frac{1}{2}\left\{\varepsilon_{\mathbf{k}}(1+B)+E_{0} \pm \sqrt{\left[\varepsilon_{\mathbf{k}}(1-B)-E_{0}\right]^{2}+4 J_{K}^{2} \lambda^{2}}\right\} .
$$

The mean-field parameters $\lambda$ and $\Gamma$ are obtained by selfconsistently solving Eqs. (4) or, equivalently, by minimizing the total internal energy

$$
E=2 \sum_{\mathbf{k}, \alpha= \pm} E_{\mathbf{k}}^{\alpha} f\left(E_{\mathbf{k}}^{\alpha}\right)+\bar{E}_{K}+\bar{E}_{H}-E_{0} N
$$

at zero temperature, or the total free energy $F$ at finite temperatures. ${ }^{17}$ The summation in Eq. (9) is made over all $\mathbf{k}$ states in the first Brillouin zone, and $f\left(E_{\mathbf{k}}\right)$ is the FermiDirac function. As usual, ${ }^{7}$ the reference energy $E_{0}$ of the $f$ band and the chemical potential $\mu$ have to be determined self-consistently in order to keep the average numbers of $f$ and conduction electrons respectively equal to 1 and $n$.

We have previously treated the half-filled case, corresponding to a number of conduction electrons $n=1,{ }^{7}$ and here we consider the general case of a noninteger number of conduction electrons. For the half-filled case, ${ }^{7}$ we have shown that the Kondo temperature $T_{K}$ for the lattice can be much smaller than the Kondo temperature $T_{K 0}$ for the single impurity, as observed in some cerium compounds. We will present in the next sections the theoretical results for bandfilling effects, and discuss later the comparison with experiment.

\section{BAND-FILLING EFFECTS AT $T=0$}

The two hybridized bands $E_{\mathbf{k}}^{ \pm}$given by Eq. (8) exhibit a structure that depends on the two factors $A \equiv J_{K}^{2} \lambda^{2}$ and $B$, defined by Eq. (7), and especially on the sign of $B$. In the case of $n=1$, previously considered, ${ }^{7}$ for small $|B|$ values such that for $|B| D^{2}<A$ there is a gap, the lower band is completely full and the upper band empty at $T=0$. For $n$ $<1$, the Fermi level cuts the lower band, and the upper band is still empty at $T=0$, which corresponds, therefore, to a real metallic situation.

The shape of the lower band $E_{\mathbf{k}}^{-}$and the solution of the case $n<1$ depend critically and self-consistently on the values of the different parameters $J_{K}, J_{H}$ and $n$ or equivalently $A, B$ and $n$. If $B>0$, i.e., $\Gamma<0$ (since $J_{H}$ is positive here), both the " $f$ band" and the hybridized lower band $E_{\mathbf{k}}^{-}$are continuously increasing with $\varepsilon_{\mathbf{k}}$. The same structure exists for $B<0$ and small $J_{H}$ values, while $E_{\mathbf{k}}^{-}$presents a maximum for $B<0$ and larger $J_{H}$ values.

Thus, the shape of $E_{\mathbf{k}}^{-}$can change under a variation of the parameters, and this peculiar situation makes the problem difficult to solve. The two characteristic cases are presented

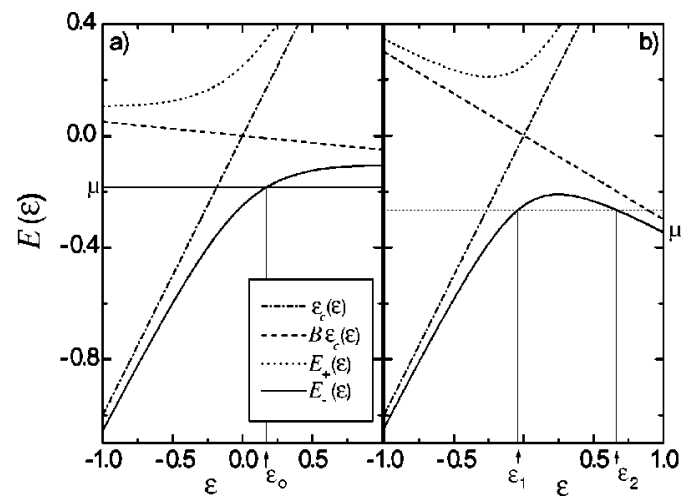

FIG. 1. Schematic plot of the nonhybridized conduction and $4 f$ bands, and of the two hybridized bands $E_{ \pm}(\mathbf{k})$. For a given $n$ $<1$, the chemical potential is different in panels (a) and (b), which correspond respectively to the two cases studied: small $J_{H}$ [a single solution of $\left.E_{-}(\varepsilon)=\mu\right]$, and large $J_{H}$ (two solutions). All energies appear in units of $D$.

schematically in Fig. 1, where we have used the fact that the energies $E_{\mathbf{k}}^{ \pm}$depend on $\mathbf{k}$ only through the bare conductionband energies $\varepsilon(\mathbf{k})$, making the substitution $E_{\mathbf{k}}^{ \pm} \rightarrow E_{ \pm}(\varepsilon)$, with $\varepsilon$ defined in the interval $[-1,1]$ in units of the half bandwidth $D$. As can be seen in Fig. 1, the curve $E_{-}(\varepsilon)$ may or may not have a maximum, according to the different cases studied. Moreover, when $E_{-}(\varepsilon)$ presents a maximum, the Fermi energy can cut the lower band in two points or in only one point within the first Brillouin zone. In fact, we have to distinguish two cases, depending on whether there are one or two intersection points [which implies automatically in the second case that the curve $E_{-}(\varepsilon)$ has a maximum]. We will compute the total energy in both situations.

For $T=0$, we firstly derive analytical expressions for both cases, and then we present numerical solutions for different sets of parameters that affect the shape of $E_{-}(\varepsilon)$. In the first case, shown in Fig. 1(a), the energy per lattice site is written as

$$
\begin{aligned}
\mathcal{E}= & \frac{1}{2 D} \int_{-D}^{\varepsilon_{0}} d \varepsilon\left\{E_{0}+(1+B) \varepsilon-\sqrt{\left[E_{0}-(1-B) \varepsilon\right]^{2}+4 J_{K}^{2} \lambda^{2}}\right\} \\
& +z J_{H} \Gamma^{2}+2 J_{K} \lambda^{2}-E_{0} .
\end{aligned}
$$

The upper limit $\varepsilon_{0}$ is the value of $\varepsilon_{\mathbf{k}}$ that corresponds to $E_{\mathbf{k}}^{-}=\mu$ in Eq. (8), as shown in Fig. 1. Writing the two selfconsistent equations that yield $n_{f}=1$ and the number of conduction electrons $n$, we obtain $\varepsilon_{0}=D n$, and

$$
E_{0}=-\frac{1}{2}(1-n)[(1-B) D+\Delta]
$$

with

$$
\Delta=\sqrt{D^{2}(1-B)^{2}+\frac{4 J_{K}^{2} \lambda^{2}}{n}} .
$$

Finally, the internal energy per site at $T=0$ [Eq. (10)] is given by 


$$
\begin{aligned}
\mathcal{E}= & z J_{H} \Gamma^{2}+2 J_{K} \lambda^{2}-E_{0}+\frac{D}{2}\left(n^{2}-1\right)-\frac{\Delta}{2} \\
& +\frac{J_{K}^{2} \lambda^{2}}{D(1-B)} \ln \left\{\frac{\Delta-D(1-B)}{\Delta+D(1-B)}\right\} .
\end{aligned}
$$

The derivatives of the total energy with respect to $\lambda$ and $\Gamma$ yield two self-consistent equations, whose solutions give the final values of $\lambda$ and $\Gamma$ corresponding to the minimum energy. For small values of $J_{H}$, Eq. (13) allows us to write

$$
\begin{gathered}
\lambda^{2}=\frac{n D^{2}(1-B)^{2}}{J_{K}^{2}} \frac{u}{(1-u)^{2}}, \\
\Gamma=\frac{n \Delta}{4 D(1-B)}-\frac{1-n}{4}-\frac{J_{K} \lambda^{2}}{D(1-B)},
\end{gathered}
$$

with $u \equiv \exp \left[-2 D(1-B) / J_{K}\right]$.

The case with a maximum in $E_{\mathbf{k}}^{-}$is more complicated. As shown in Fig. 1, for the case $B<0$ with large values of $J_{H}$, the Fermi level $\mu$ cuts the band $E_{\mathbf{k}}^{-}$in two energies; then, the two corresponding energies $\varepsilon_{1}$ and $\varepsilon_{2}$ are given by the values of $\varepsilon(\mathbf{k})$ which are roots of the equation $E_{\mathbf{k}}^{-}=\mu$ with $E_{\mathbf{k}}^{-}$ given by eq.(8). So, the expression of the energy is given by Eq. (10) by changing the integration limits so that the integral is now performed from $-D$ to $\varepsilon_{1}$ and from $\varepsilon_{2}$ to $D$.

Now, the two equations derived from the constraint $n_{f}$ $=1$ and the number of conduction electrons equal to $n$ are

$$
\begin{gathered}
\varepsilon_{2}-\varepsilon_{1}=D(1-n), \\
E_{0}=-(1-n) \sqrt{D^{2}+\frac{4 J_{K}^{2} \lambda^{2}}{(1-B)^{2}-(1-n)^{2}}} .
\end{gathered}
$$

We can derive analytical expressions for $\varepsilon_{1}$ and $\varepsilon_{2}$, and finally for the total energy per site $\mathcal{E}$ for $B<0$. A quite long calculation yields

$$
\begin{aligned}
\mathcal{E}= & -\frac{R}{2(1-B)}\left[(1-B)^{2}-2 B(1-n)+\left(1-n^{2}\right)\right] \\
& -\frac{J_{K}^{2} \lambda^{2}}{(1-B) D} \ln \left\{\frac{P-D(1-n) \sqrt{|B|}}{P+D(1-n) \sqrt{|B|}} \frac{R+D}{R-D}\right\} \\
& +\frac{1-n}{2(1-B)} P \sqrt{|B|}+\frac{D^{2} B^{2}}{z J_{H}}+2 J_{K} \lambda^{2}-E_{0},
\end{aligned}
$$

where

$$
R=\sqrt{D^{2}+\frac{4 J_{K}^{2} \lambda^{2}}{(1-B)^{2}-(1-n)^{2}}}
$$

and

$$
P=\sqrt{4 J_{K}^{2} \lambda^{2}-D^{2} B(1-n)^{2}} .
$$

Minimizing the energy (18) with respect to $\lambda$ we obtain

$$
\lambda^{2}=p / 2 a,
$$

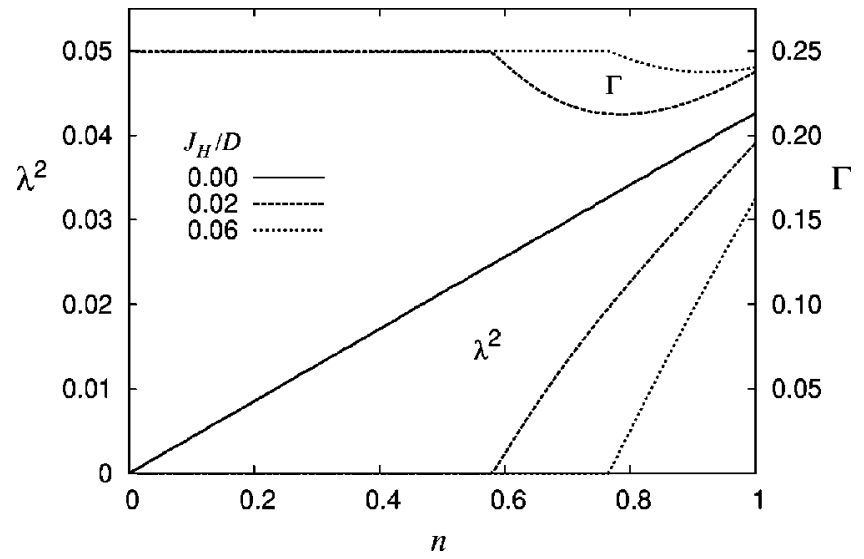

FIG. 2. Variation of $\lambda^{2}$ and $\Gamma$ with the band filling $n$ for $J_{K} / D=0.4$ and different values of $J_{H}$.

with

$$
p=2 J_{K}-\frac{J_{K}^{2}}{D(1-B)} \ln \left[\frac{(1-B)^{2}-(1-n)^{2}}{-B(1-n)^{2}}\right]
$$

and

$$
a=\frac{J_{K}^{2}}{4 D(1-B)}\left[\frac{1}{B(1-n)^{2}}+\frac{1}{(1-B)^{2}-(1-n)^{2}}\right] .
$$

For the present case, $\lambda$ vanishes at a critical band filling given by

$$
n_{c}=1-(1-B) \sqrt{\frac{u}{u-B}} .
$$

We have finally performed a numerical calculation by considering the general form of the energy given by either Eq. (10) or Eq. (18) in the different cases studied, and looking for the minimum energy of the system. In fact, for each value of the different parameters, we compare the two solutions and we retain the lowest-energy one, thus determining the corresponding $\lambda$ and $\Gamma$.

Figure 2 shows $\lambda^{2}$ and $\Gamma$ versus $n$ for different values of $J_{H}$, and $J_{K} / D=0.4$. For $J_{H}=0$ we obtain negative values of $\Gamma$ (not shown in Fig. 2), with $\Gamma \rightarrow-1 / 4$ in the limit $n \rightarrow 0$ [see Eq. (15)]. This is consistent with previous results ${ }^{17,29}$ showing that $\Gamma$ changes sign as a function of $n$ when the Kondo effect is strongly dominant.

Figure 3 shows the phase diagram for $J_{K} / D=0.4$ and 1.0. In the latter case (previously reported in Ref. 29), for small $J_{H}$ the Kondo phase is stable for all values of the band filling, while for large $J_{H}$ the Kondo phase is stable only for $n>n_{c}$ given by Eq. (24). Figure 3 also shows that Eq. (24) is very close to the numerical result in this region, but does not apply when $J_{H}$ is very small, since the assumed maximum in the lower energy band no longer exists. The crossover between these two regimes was obtained numerically. In the crossover region we found a discontinous transition from $\lambda$ $\neq 0$ to $\lambda=0$. These results have to be taken with caution, however, since the value of $J_{K} / D=1$ is unphysically high, 


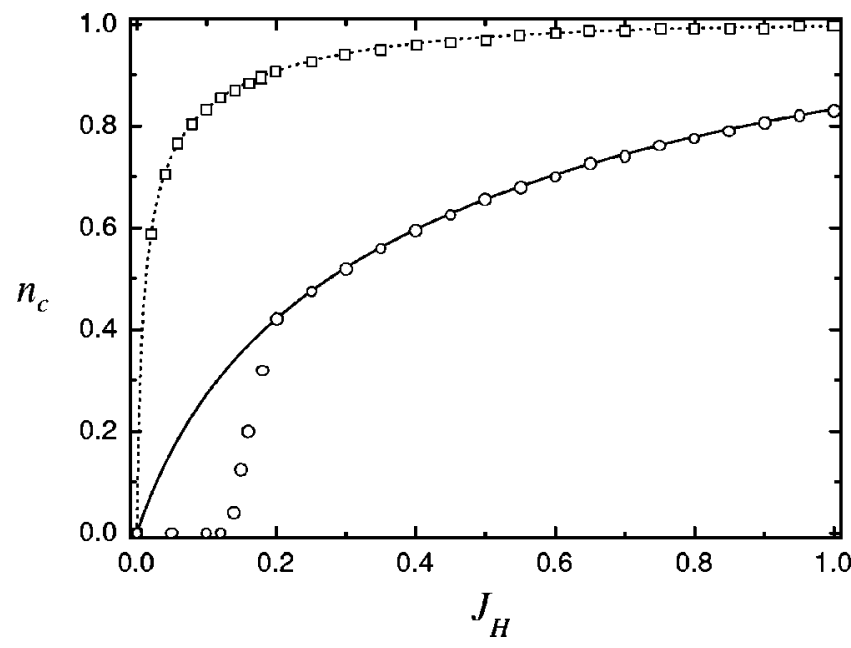

FIG. 3. Phase diagram plotted as the critical band filling $n_{c}$ versus $J_{H}$. The curves are drawn from Eq. (24) for $J_{K} / D=0.4$ (dotted line) and 1.0 (solid line). The symbols correspond to the results obtained by minimizing the energy. In each case, the Kondo regime is stable above the line, and the magnetic phase below.

and the Kondo lattice is expected to show ferromagnetic behavior in the low- $n$ limit, ${ }^{22}$ while our analysis is restricted to antiferromagnetic intersite exchange.

Thus, our calculation shows clearly that small $n$ and large $J_{H}$ values tend to suppress the Kondo effect, yielding a "magnetic" phase with $\lambda=0$ and large short-range magnetic correlations. In fact, in this region a long-range magnetic order should certainly be stabilized, but this was not taken into account in this approach. In contrast, both $\lambda$ and $\Gamma$ are different from zero in the Kondo phase.

\section{BAND-FILLING EFFECTS AT FINITE TEMPERATURE}

Focusing on the Kondo-lattice problem at finite temperatures, the half-filled case has been previously described, and we consider here the general case of $n<1$. The number of $f$ electrons $n_{f}$ is always taken equal to 1 . The values of $\lambda$ and $\Gamma$ are determined by self-consistently solving Eqs. (4) or by minimizing the free energy, which is given by

$$
F=-2 T \sum_{k, \alpha= \pm} \ln \left[1+e^{-\left(E_{\alpha}-\mu\right) / T}\right]-E_{0}+z J_{H} \Gamma^{2}+2 J_{K} \lambda^{2} .
$$

In our mean-field approximation, $T_{K}$ and $T_{\text {cor }}$ are defined as the temperatures at which, respectively, $\lambda$ and $\Gamma$ become zero. We have shown, in the case $n=1$, that the Kondo temperature $T_{K}$ for the lattice can be much smaller than the Kondo temperature $T_{K 0}$ for the single impurity, as observed in some cerium compounds. ${ }^{7}$ Moreover, we have also shown that the temperature $T_{\text {cor }}$ for the occurrence of short-range magnetic correlations lies above the Kondo temperature $T_{K}$ for large $J_{H}$ values, while $T_{\text {cor }}=T_{K}$ for small $J_{H}$. These two theoretical results have provided a good explanation for the previously described anomalous behavior observed in some cerium compounds.

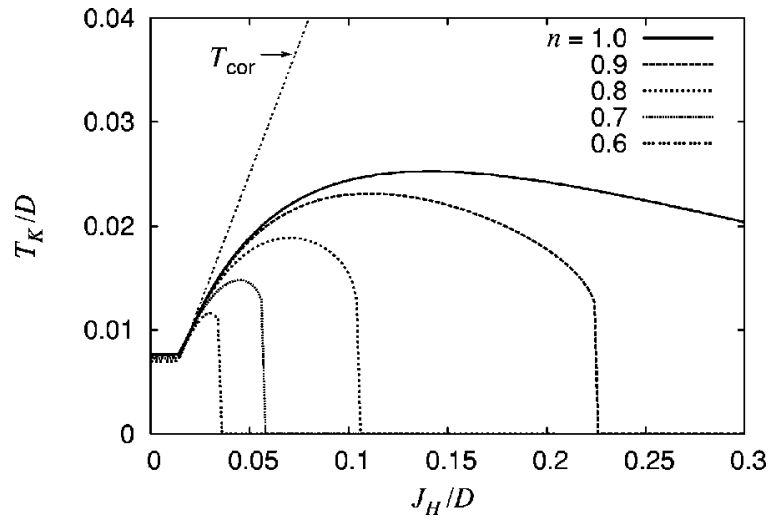

FIG. 4. Kondo temperature $T_{K}$ versus $J_{H}$ for $J_{K} / D=0.4$ and several values of $n$. We also show the correlation temperature $T_{\text {cor }}$.

First, we present analytical results obtained in the case of large $J_{H}$ values, where $T_{\text {cor }}>T_{K}$. In this case, $T_{\text {cor }}$ is easily determined by taking the limit $\Gamma \rightarrow 0$ in the self-consistent equation for $\Gamma$ with fixed $\lambda=0$, and we obtain

$$
T_{\text {cor }}={ }_{z} J_{H} / 12 \text {, }
$$

where $z$ is the number of nearest neighbors. In the simple cubic case, $z=6$, we obtain $T_{\text {cor }}=J_{H} / 2$. Still for the case $T_{\text {cor }}>T_{K}$, we determine $T_{K}$ by taking the limit $\lambda \rightarrow 0$ in the self-consistent equation for $\lambda$, with $\Gamma$ remaining finite. We obtain the following equation to determine $T_{K}$ :

$$
\begin{aligned}
\frac{1}{\rho J_{K}}= & \int_{-D}^{-D} d \varepsilon\left[\frac{1}{\varepsilon(1-B)-E_{0}}\left(\frac{1}{1+e^{(\varepsilon-\mu) / T_{K}}}\right)\right] \\
& \left.\left.-\frac{1}{1+e^{\left(E_{0}+B \varepsilon-\mu\right) / T_{K}}}\right)\right]
\end{aligned}
$$

Equation (27), together with the self-consistent evaluation of $B$ [see Eqs. (7) and (4)], and the two equations giving $n_{f}$ $=1$ and $n<1$ (which yield $E_{0}$ and $\mu$ ) allow us to determine $T_{K}$. In the case of $J_{H}=0$, we recover the simple equation

$$
\frac{2}{\rho J_{K}}=\int_{-D}^{-D} d \varepsilon\left[\frac{1}{\varepsilon-E_{0}} \tanh \left(\frac{\varepsilon-\mu}{2 T_{K}}\right)\right],
$$

which reproduces the single-impurity result. In the limit $J_{K} / D \ll 1$, this yields

$$
T_{K}=C_{0} D \sqrt{n(2-n)} \exp \left(-\frac{1}{\rho J_{K}}\right),
$$

where $C_{0}$ is a numerical constant: $C_{0}=1.1337$. Equation (29) gives $T_{K}$ depending on the band filling as $\sqrt{n(2-n)}$, which is consistent with a $\sqrt{n}$ dependence when $n \rightarrow 0$, as found in Ref. 23.

Now, we present numerical results obtained at finite temperatures in the case $n<1$, for different sets of parameters $J_{K}, J_{H}$, and $n$, in order to study the Kondo-lattice properties and band-filling effects. Figure 4 gives the curves of the Kondo temperature $T_{K}$ versus $J_{H}$ for a given $J_{K}$ value and several values of the conduction-band filling $n$. We see 


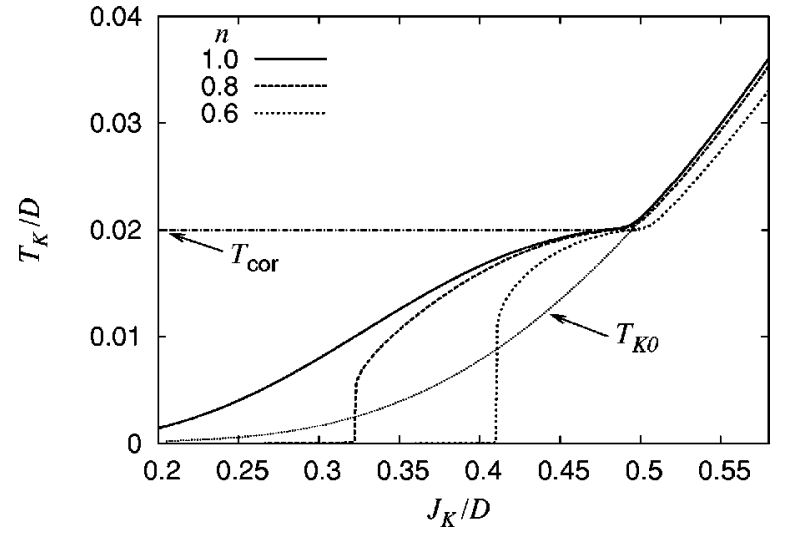

FIG. 5. Kondo temperature $T_{K}$ as a function of $J_{K}$ for $J_{H} / D$ $=0.04$ and representative values of $n$. We also show the correlation temperature $T_{\text {cor }}$, and the single-impurity Kondo temperature $T_{\mathrm{K} 0}$.

clearly that $T_{K}$ first increases, and then decreases with $J_{H}$ for fixed $n$, dropping abruptly to zero at some critical value of $J_{H}$. On the other hand, for a given $J_{H}, T_{K}$ decreases rapidly as $n$ departs from half filling. The enhancement of $T_{K}$ for small $J_{H}$ was masked in Ref. 7 by the choice of $J_{H}=\alpha J_{K}^{2}$, which intended to mimic the RKKY interaction. Besides this being a questionable approximation, we have preferred here to study the behavior of the Kondo temperature as a function of $J_{K}$ and $J_{H}$ considered as independent parameters. For comparison, in Fig. 4 we have also plotted the correlation temperature $T_{\text {cor }}$, which is linear with $J_{H}$ independently of the value of $n{ }^{7}$ Figure 5 gives $T_{K}$ as a function of $J_{K}$ for $J_{H} / D=0.04$ and representative values of $n$. Here again we include the correlation temperature $T_{\text {cor }}$, which signals the onset of short-range magnetic correlations when the temperature is lowered at fixed $J_{K}$. For comparison, we plot the single-impurity Kondo temperature $T_{K 0}$ (for $\left.n \leq 1\right)$, which varies exponentially with $J_{K}$, and is weakly dependent on $n$ near half-filling [see Eq. (29)].

Figures 4 and 5 show some interesting results of our model. First, one can see the occurrence of short-range magnetic correlations above the Kondo temperature, in good agreement with experiment. Also, in the region of coexistence between Kondo effect and magnetic correlations, the Kondo temperature is enhanced with respect to the singleimpurity case, and shows a smoother variation with $J_{K}$. Such an enhancement agrees with the idea ${ }^{30}$ that $f$ - $f$ correlations may, in some cases, reinforce the Kondo effect on a lattice. The second noticeable feature of Figs. 4 and 5 is the almost catastrophic suppression of the Kondo effect with increasing intersite coupling, and the enhancement of this behavior as the band-filling factor is reduced.

Now, in order to better understand the physics of the Kondo lattice at low temperatures, and to see more clearly the different regimes occurring with decreasing temperature, we present different curves showing the product of magnetic susceptibility and temperature, $\chi T$, which represents the square of the effective magnetic moment.

On the two following figures, $\chi T$ is constant at high temperatures down to a first kink corresponding to either the correlation temperature $T_{\text {cor }}$ for a sufficiently large $J_{H}$ value

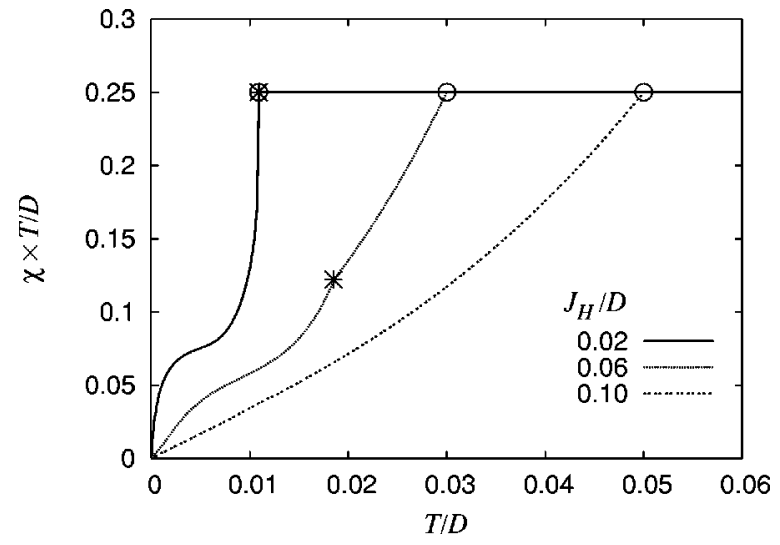

FIG. 6. Magnetic susceptibility times temperature as a function of temperature for $n=0.8, J_{K} / D=0.4$, and different values of $J_{H}$. The open circles mark the position of $T_{\text {cor }}$, and the stars, that of $T_{K}$. These two temperatures coincide in the case of $J_{H} / D=0.02$.

or to $T_{K}$ at low $J_{H}$ values. The kink corresponding to $T_{K}$ in the case when $T_{K}<T_{\text {cor }}$ is less pronounced, but still clearly visible. Figure 6 gives the results for the temperature dependence of the product $\chi T$ for $J_{K} / D=0.4, n=0.8$, and representative values of $J_{\mathrm{H}}$. This figure shows a Kondo regime alone for small $J_{H}$, a regime with only short-range magnetic correlations above the Kondo regime for intermediate $J_{H}$, and the absence of the Kondo phase for large $J_{H}$. Then, Fig. 7 gives the results for the temperature dependence of the product $\chi T$ for $J_{K} / D=0.4$, an intermediate value $J_{H} / D$ $=0.04$, and representative values of $n$. The curves show the onset of short-range correlations and the Kondo regime for $n$ values not very far from 1, while the Kondo effect has completely disappeared for $n=0.6$, in which case a single critical temperature remains. We immediately see on this figure that the behavior of the magnetic susceptibility is completely different for $n=1$ and smaller $n$ values, because the $n=1$ case corresponds to an insulating system, and smaller $n$ values yield metallic behavior. We also see that the Kondo effect disappears when $n$ decreases below a critical value, which

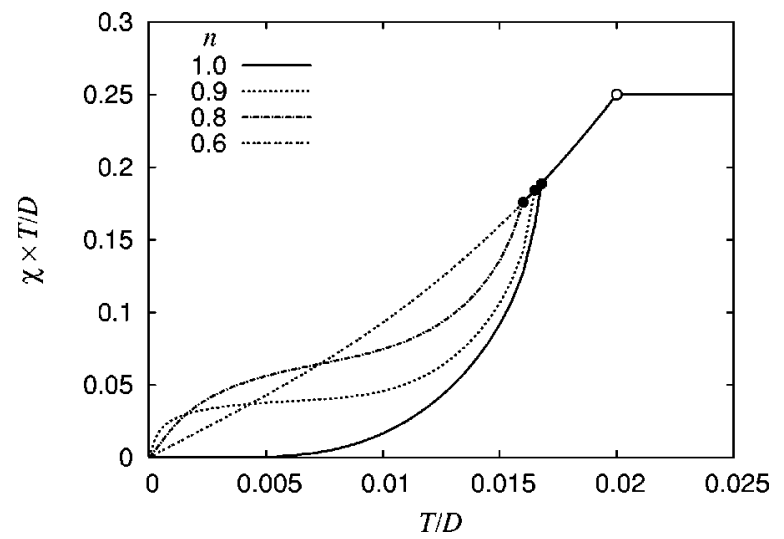

FIG. 7. Magnetic susceptibility times temperature as a function of temperature for $J_{K} / D=0.4, J_{H} / D=0.04$, and different values of $n$. The open circle signals $T_{\text {cor }}$, while the filled circles indicate the positions of $T_{K}$ for the three higher values of $n$ (the Kondo regime no longer occurs for $n=0.6$ ). 


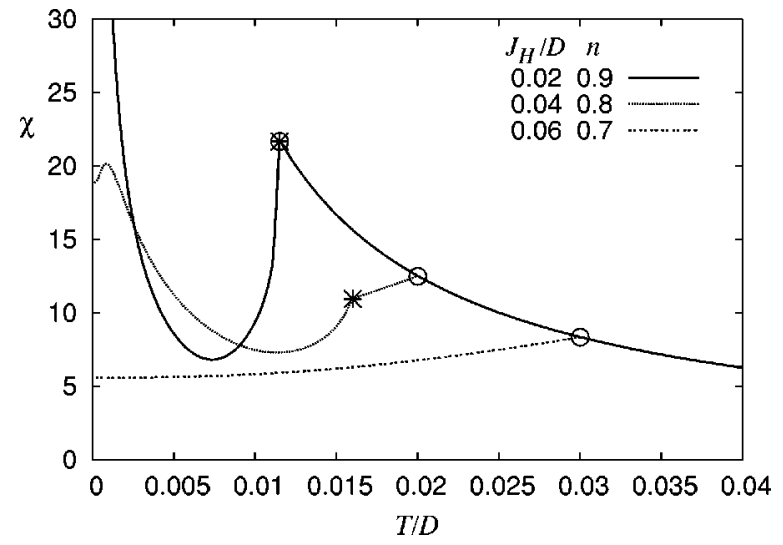

FIG. 8. Magnetic susceptibility as a function of temperature for $J_{K} / D=0.4$ and different sets of the parameters $J_{H}$ and $n$, in order to show the different regimes. Circles and stars follow the convention of Fig. 6.

can be interpreted as a manifestation of the "exhaustion" problem $^{16}$ in connection with the competition between Kondo effect and magnetic correlations.

Finally, in Fig. 8, we present a plot of the magnetic susceptibility versus temperature for the three different regimes for typical sets of parameters: Kondo regime alone (small $J_{H}, n$ near half-filling), both magnetic correlations and Kondo regimes (higher $J_{H}$, smaller $n$ ), and short-range magnetic correlations only (still higher $J_{H}$, relatively small $n$ ). This figure gives an illustrative summary of the physics of the Kondo-lattice model.

The different behaviors shown in the last three figures are worth analyzing in more detail. At high temperatures, the magnetic susceptibility is decreasing in $1 / T$ as normally expected. Then, below the highest characteristic temperature $\left(T_{\text {cor }}\right.$ or $T_{K}$ ), there is a rapid decrease of the product $\chi T$, indicating a reduction of the effective magnetic moment due to the occurence of short-range antiferromagnetic correlations, which tend to reduce the response of the localized moments to an applied magnetic field, and an even faster reduction due to the Kondo effect. At lower temperatures, within the Kondo regime, we observe a flattening of the effective magnetic moment. This region of nearly constant magnetic moment below $T_{K}$ can be related to the "exhaustion" problem, because there are not enough electrons to screen the localized spins. At still lower temperatures, a coherent regime sets in, and this residual moment becomes completely screened. This behavior is not observed in the half-filled case, where the effective magnetic moment tends very rapidly to zero due to the existence of a gap at the Fermi level.

\section{CONCLUSIONS}

We have studied the different behaviors of the intrasite and intersite correlations, and the characteristic temperatures as a function of the intrasite Kondo exchange coupling, of the intersite exchange coupling, and of the number of conduction electrons relative to the number of $f$ electrons, which remains fixed to 1 . We use here a mean-field approximation with two order parameters in each case, the mean-field Kondo correlator and the short-range magnetic correlation.

We have established here for $n<1$, as in previous work ${ }^{7}$ for $n=1$, that the dependence of the Kondo temperature with the coupling constant $J_{K}$ for the lattice can be significantly different from the single-impurity case. This result can account for the pressure dependence of $T_{K}$ observed in $\mathrm{CeRh}_{2} \mathrm{Si}_{2},{ }^{3} \mathrm{CeRu}_{2} \mathrm{Ge}_{2},{ }^{5,6}$ or more recently $\mathrm{Ce}_{2} \mathrm{Rh}_{3} \mathrm{Ge}_{5} .{ }^{8}$ On the other hand, depending on the relative values of $J_{H}$ and $J_{K}$, as well as on the band-filling, the lattice Kondo temperature can closely follow the single-impurity one, as observed in many cerium and all ytterbium compounds. Further experiments are needed to better understand the conditions yielding a Kondo temperature for the lattice much different than the single-impurity one. This issue has also been addressed by different theoretical approaches to both the Kondo lattice and the periodic Anderson model. ${ }^{21,23,25-27,31}$

Another interesting result concerns the derivation of a correlation temperature below which short-range magnetic correlations appear, in good agreement with neutron scattering experiments in cerium compounds. These correlations can coexist with the Kondo effect, but eventually dominate, and suppress the Kondo regime for sufficiently high values of the intersite exchange interaction or sufficiently low band fillings.

Our present calculation addresses again the difficult issue of the nature of the ground state and screening in the Kondolattice problem. We have shown here that, as the number of conduction electrons is reduced, exhaustion may be compensated by formation of intersite singlets of localized spins. Exact calculations for small clusters would be interesting in that a comparison between results with a number of conduction electrons equal to, or much smaller than the number of $4 f$ electrons localized on the different sites of the clusters could shed new light on this issue. ${ }^{32}$ Finally, it is interesting to notice that taking into account lattice effects is essential for describing the properties of cerium or other anomalous rare-earth compounds at low temperatures, as shown, for example, in photoemission experiments. ${ }^{27,28}$
${ }^{1} \mathrm{~S}$. Doniach, Proceedings of the International Conference on Valence Instabilities and Related Narrow-Band Phenomena, edited by R. D. Parks (Plenum Press, New York, 1976), p. 168.

${ }^{2}$ B. Barbara, H. Bartholin, D. Florence, M. F. Rossignol, and E. Walker, Physica B 86-88, 188 (1977).
${ }^{3}$ T. Graf, J. D. Thompson, M. F. Hundley, R. Movshovich, Z. Fisk, D. Mandrus, R. A. Fischer, and N. E. Phillips, Phys. Rev. Lett. 78, 3769 (1997).

${ }^{4}$ K. Alami-Yadri, H. Wilhelm, and D. Jaccard, Physica B 259-261, 157 (1999). 
${ }^{5}$ S. Sullow, M. C. Aronson, B. D. Rainford, and P. Haen, Phys. Rev. Lett. 82, 2963 (1999).

${ }^{6}$ H. Wilhelm, K. Alami-Yadri, B. Revaz, and D. Jaccard, Phys. Rev. B 59, 3651 (1999).

${ }^{7}$ J. R. Iglesias, C. Lacroix, and B. Coqblin, Phys. Rev. B 56, 11820 (1997).

${ }^{8}$ K. Umeo, T. Takabatake, T. Suzuki, S. Hane, H. Mitamura, and T. Goto, Phys. Rev. B 64, 144412 (2001).

${ }^{9}$ J. Rossat-Mignod, L. P. Regnault, J. L. Jacoud, C. Vettier, P. Lejay, J. Flouquet, E. Walker, D. Jaccard, and A. Amato, J. Magn. Magn. Mater. 76-77, 376 (1988).

${ }^{10}$ J. Pierre, P. Haen, C. Vettier, and S. Pujol, Physica B 163, 463 (1990).

${ }^{11}$ J. Flouquet, S. Kambe, L. P. Regnault, P. Haen, J. P. Brison, F. Lapierre, and P. Lejay, Physica B 215, 77 (1995).

${ }^{12}$ L. P. Regnault, W. A. C. Erkelens, J. Rossat-Mignod, P. Lejay, and J. Flouquet, Phys. Rev. B 38, 4481 (1988).

${ }^{13}$ J. M. Mignot, J. L. Jacoud, L. P. Regnault, J. Rossat-Mignod, P. Haen, P. Lejay, P. Boutrouille, B. Hennion, and D. Petitgrand, Physica B 163, 611 (1990).

${ }^{14}$ L. P. Regnault, J. L. Jacoud, J. M. Mignot, J. Rossat-Mignod, C. Vettier, P. Lejay, and J. Flouquet, Physica B 163, 606 (1990).

${ }^{15}$ C. Lacroix and M. Cyrot, Phys. Rev. B 20, 1969 (1979).

${ }^{16}$ P. Nozières, Eur. Phys. J. B 6, 447 (1998).

${ }^{17}$ A. R. Ruppenthal, J. R. Iglesias, and M. A. Gusmão, Phys. Rev. B 60, 7321 (1999).

${ }^{18}$ B. Coqblin, M. A. Gusmão, J. R. Iglesias, C. Lacroix, A. Ruppenthal, and Acirete S. Da R. Simoes, Physica B 281-282, 50 (2000)
${ }^{19}$ B. Coqblin, M. A. Gusmão, J. R. Iglesias, A. Ruppenthal, and C. Lacroix, J. Magn. Magn. Mater. 226-230, 115 (2001).

${ }^{20}$ R. Jullien and P. Pfeuty, J. Phys. F: Met. Phys. 11, 353 (1981).

${ }^{21}$ M. A. Continentino, G. M. Japiassu, and A. Troper, Phys. Rev. B 39, 9734 (1989).

${ }^{22}$ H. Tsunetsugu, Y. Hatsugai, K. Ueda, and M. Sigrist, Phys. Rev. B 46, 3175 (1982).

${ }^{23}$ S. Burdin, A. Georges, and D. R. Grempel, Phys. Rev. Lett. 85, 1048 (2000).

${ }^{24}$ S. Burdin, thesis, Grenoble (2001).

${ }^{25}$ A.H. Castro Neto, and B.A. Jones, Phys. Rev. B 62, 14975 (2000).

${ }^{26}$ B. H. Bernhard, C. Lacroix, J. R. Iglesias, and B. Coqblin, Phys. Rev. B 61, 441 (2000).

${ }^{27}$ A. N. Tahvildar, M. Jarrell, and J. K. Freericks, Phys. Rev. B 55, R3332 (1997).

${ }^{28}$ A. J. Arko, J. J. Joyce, A. B. Andrews, J. D. Thompson, J. L. Smith, D. Mandrus, M. F. Hundley, A. L. Cornelius, E. Moshopoulou, Z. Fisk, P. C. Canfield, and Alois Menovsky, Phys. Rev. B 56, R7041 (1997).

${ }^{29}$ C. Lacroix, J. R. Iglesias, and B. Coqblin, Physica B 312-313, 159 (2002).

${ }^{30}$ P. Coleman and N. Andrei, J. Phys.: Condens. Matter 1, 4057 (1989).

${ }^{31}$ H. Tsunetsugu, M. Sigrist, and K. Ueda, Rev. Mod. Phys. 69, 809 (1997).

${ }^{32}$ C. Busser, E. V. Anda, J. R. Iglesias, and B. Coqblin, J. Magn. Magn. Mater. 226-230, 134 (2001). 